\title{
Dickschicht-Sensor zur Detektion von Wasserstoff in Chlorgas
}

\author{
Stefan Dietrich $^{1}$, Mihails Kusnezoff', Nikolai Trofimenko ${ }^{1}$, Wieland Beckert ${ }^{1}$, Jost Henze ${ }^{2}$ \\ ${ }^{1}$ Fraunhofer-IKTS, Winterbergstr. 28, 01277 Dresden, Deutschland, stefan.dietrich@ikts.fraunhofer.de \\ 2 Henze-Hauck Analytik GmbH, Dünnhauptstr. 14, 06847 Dessau-Rosslau, Deutschland
}

\begin{abstract}
Kurzfassung
Es wurde ein Sensor zur Detektion von Wasserstoff in Chlorgas auf Basis keramischer Dickschichttechnologie entwickelt. Der auf dem Wärmetönungsprinzip beruhende Sensor zeigt ein schnelles Ansprechverhalten auf Wasserstoffkonzentrationen $\geq 1$ Vol.- $\%$ in feuchtem Chlorgas und eine ausgezeichnete Langzeitstabilität in korrosiver Gasatmosphäre.
\end{abstract}

\begin{abstract}
A sensor for the detection of hydrogen in chlorine has been developed. Based on ceramic thick film technology, the sensor features fast response to hydrogen concentrations of $\geq 1 \% / v o l$. in damp chlorine and excellent long term stability in corrosive gas environment.
\end{abstract}

Key words: Gassensor, Wasserstoff, Chlorgas, Dickschicht, Chemische Industrie

\section{Einleitung}

Chlor ist eine der wichtigsten und meistproduzierten Grundchemikalien, seine großtechnische Herstellung erfolgt überwiegend auf dem Wege der Chloralkalielektrolyse. Der bei diesem Verfahren neben Chlor entstehende Wasserstoff birgt ein großes Gefahrenpotential für Mensch, Umwelt und Produktionsanlagen, da die bei Undichtheiten in der Anlage mögliche Chlorknallgasreaktion stark exotherm verläuft und bereits durch Belichtung oder Wärmezufuhr initiiert werden kann. Um Explosionen in der Produktionsanlage zu vermeiden, ist die Trennung beider Gase in der Prozessführung daher von großer Bedeutung. Zur Detektion von Wasserstoff in Chlorgas existieren zwar kommerzielle Sensorlösungen, jedoch ist deren Anschaffung und Betrieb in der Regel sehr kostenintensiv.

Am Fraunhofer-IKTS wurde deshalb gemeinsam mit der Henze-Hauck Prozessmesstechnik und Analytik $\mathrm{GmbH}$ ein auf dem Wärmetönungsprinzip basierender Sensor entwickelt, welcher die kontinuierliche Bestimmung der Wasserstoffkonzentration im Chlorgas erlaubt. Die im Sensor eingesetzten Heizelemente werden in keramischer Dickschichttechnologie gefertigt und zeichnen sich neben der kostengünstigen Herstellung vor allem durch eine hohe Langzeitstabilität in korrosiver Gasatmosphäre aus.

\section{Werkstoffauswahl und -testung}

Beim Einsatz unter Produktionsbedingungen stehen die Sensorelemente in direktem Kontakt mit dem bis zu $80{ }^{\circ} \mathrm{C}$ heißen und $100 \%(\mathrm{RH})$ feuchten Chlorgas, woraus hohe Anforderungen an die Korrosionsbeständigkeit erwachsen. Als mögliche Werkstoffkategorien für die Herstellung der Heizelemente kommen vor allem Aluminiumoxid $\left(\mathrm{Al}_{2} \mathrm{O}_{3}\right)$ und Zirkoniumdioxid $\left(\mathrm{ZrO}_{2}\right)$ mit unterschiedlichen Dotierungen, technische Gläser sowie Edelmetalle, hier v.a. Platin, in Betracht. Zu Beginn der Entwicklung waren zu einigen dieser Werkstoffe zwar wissenschaftliche Arbeiten zur Beständigkeit in sauren und basischen Medien sowie unter hydrothermalen Bedingungen, nicht aber zum Verhalten in feuchtem Chlorgas zu finden. So fanden sich Hinweise, dass bei technischen Gläsern beispielsweise jene mit Anteilen von $\mathrm{PbO}$ und $\mathrm{ZnO}$ eine geringe Korrosionsstabilität zeigen, während die Additive $\mathrm{Al}_{2} \mathrm{O}_{3}, \mathrm{Si}_{3} \mathrm{~N}_{4}$ und $\mathrm{TiO}_{2}$ in geeigneten Mengen zu einer erhöhten Säureresistenz der Gläser führen [1-4].

Für Zirkoniumdioxid empfiehlt sich meist der Einsatz verschiedener Dotierungen, um die sonst nur bei Temperaturen von ca. $2700{ }^{\circ} \mathrm{C}$ vorliegende kubische Phase zumindest partiell auch bei niedrigen Temperaturen zu stabilisieren. Insbesondere in korrosiven Medien kommt dieser Stabilisierung und dem dafür eingesetzten Metalloxid eine noch größere Bedeutung zu: Die korrosive Umgebung kann 
hier andernfalls zu einer Umwandlung tetragonaler Phasenanteile in die monokline Phase führen, was mit einer Volumenexpansion und einer damit verbundenen Rissbildung einhergeht. Verschiedene Literaturquellen deuten an, dass zumindest eine partielle Stabilisierung in der kubischen Phase die Korrosionsbeständigkeit erhöhen kann [5,6]. $\mathrm{Sc}_{2} \mathrm{O}_{3}$ wie auch $\mathrm{Y}_{2} \mathrm{O}_{3}$ erweisen sich hier als geeignete Dotanten.

Beim Einsatz von Platin in Chlorgas ist zu beachten, dass ab einer Temperatur von ca. $550{ }^{\circ} \mathrm{C}$ die Bildung von Platin(II)-Chlorid möglich ist.

In Voruntersuchungen wurde die Korrosionsbeständigkeit ausgewählter Werkstoffe beurteilt. Die Auswahl umfasste $120 \mu \mathrm{m}$ dicke Proben kommerzieller Keramiksubstrate (Fa. Kerafol, Fa. ESL) aus Zirkoniumdioxid, welches mit 3, 5, und $8 \mathrm{Mol}-\% \mathrm{Y}_{2} \mathrm{O}_{3}$ dotiert ist (nYSZ, $n=\{3 ; 5 ; 8\}$ ) sowie Substratproben aus 3YSZ, welche mit einer kommerziell erhältlichen Platin-Dickschichtpaste (LPA 88-11 S, Fa. Heraeus) bedruckt und gesintert wurden. Darüber hinaus wurden Proben aus Quarzglas sowie Platindrähte in die Voruntersuchungen einbezogen.

Die ersten Auslagerungsuntersuchungen erfolgten in Salzsäure-Dampf $(\mathrm{HCl})$ in einem Autoklaven (Fa. Berghof), welcher mit $\mathrm{HCl}$ $(10 \mathrm{~m} \%)$ teilbefüllt und auf $150{ }^{\circ} \mathrm{C}$ beheizt wurde, der Dampfdruck betrug ca. 4 bar. Die keramischen Proben mit einer Größe von ca. $3 \times 30 \times 0,12 \mathrm{~mm}^{3}$ wurden hierzu zunächst im Ultraschallbad (Ethanol) gereinigt, bei $120{ }^{\circ} \mathrm{C}$ in einem Trockenschrank getrocknet, mit einer Auflösung von $10 \mu \mathrm{g}$ auf einer Analysenwaage gewogen (Fa. Mettler Toledo) und anschließend in eine Teflon-Halterung im Autoklaven überführt. Ebenso wurde mit den Quarzglasproben sowie den Platindrähten verfahren. Durch die Teilbefüllung des Autoklaven wurde sichergestellt, dass die Proben während der Auslagerung nicht unmittelbar der Salzsäure, sondern lediglich dem HCl-Dampf ausgesetzt sind. Nach der Auslagerung über eine Dauer von $150 \mathrm{~h}$ wurden die Proben sofern intakt - auf dieselbe Weise gereinigt, getrocknet und gewogen. Abb. 1 zeigt eine Fotographie der Reste der 3- und 5YSZSubstratproben, welche bei der Auslagerung vollständig zerstört wurden.

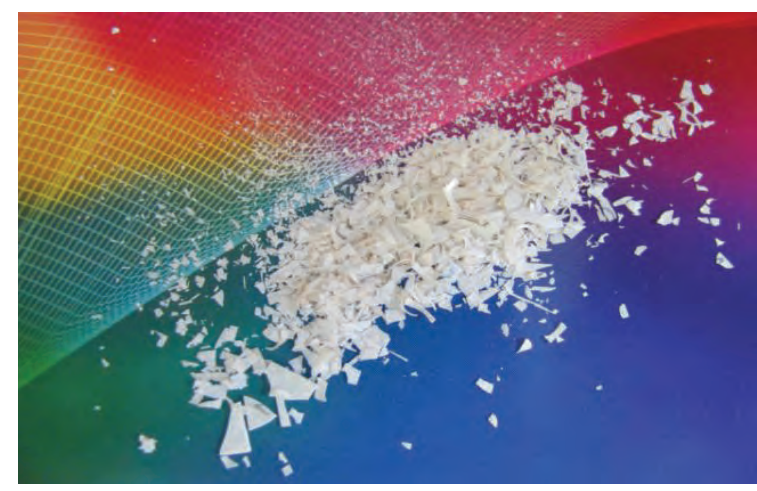

Abb. 1. Bei Auslagerung in HCl-Dampf zerstörte 3und 5YSZ-Substratproben.

In Abb. 2 ist eine FESEM-Aufnahme der Oberfläche eines ausgelagerten 3YSZSubstratfragments dargestellt.

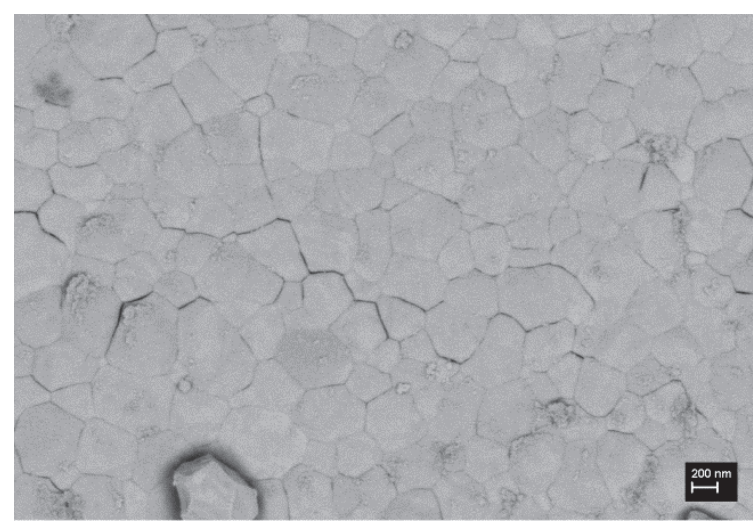

Abb. 2. FESEM-Aufnahme der Oberfläche eines ausgelagerten 3YSZ-Substratfragments.

Deutlich sind hier Mikrorisse entlang der Korngrenzen zu erkennen, welche in Summe zur Zerstörung der Probe beigetragen haben. Lediglich die vollständig in der kubischen Phase stabilisierten 8YSZ-Proben konnten dem Autoklaven intakt und ohne signifikanten Massenverlust entnommen werden.

In Abb. 3a und $b$ sind FESEM-Aufnahmen der Probenoberfläche der Platin-Dickschichtstruktur einer nicht ausgelagerten Referenzprobe (a) und einer Probe nach Auslagerung (b) abgebildet. Gut zu erkennen ist hier die deutlich erhöhte Porosität der ausgelagerten Probe. Die Erklärung für diese Degradation konnte anhand einer vergleichenden EDX-Elementanalyse von Referenz- und ausgelagerten Proben gefunden werden: Die gesinterte kommerzielle Pt-Paste der Referenzprobe enthält als Strukturbildner demnach Anteile eines Barium-haltigen Glases. $\mathrm{Da}$ in der EDX-Analyse der ausgelagerten Proben keine Hinweise mehr auf Barium gefunden werden konnten, ist anzunehmen, dass dieses während der Auslagerung zu einem erheblichen Teil aus dem Glas gelöst wurde. Dies hatte offenbar die weitgehende 


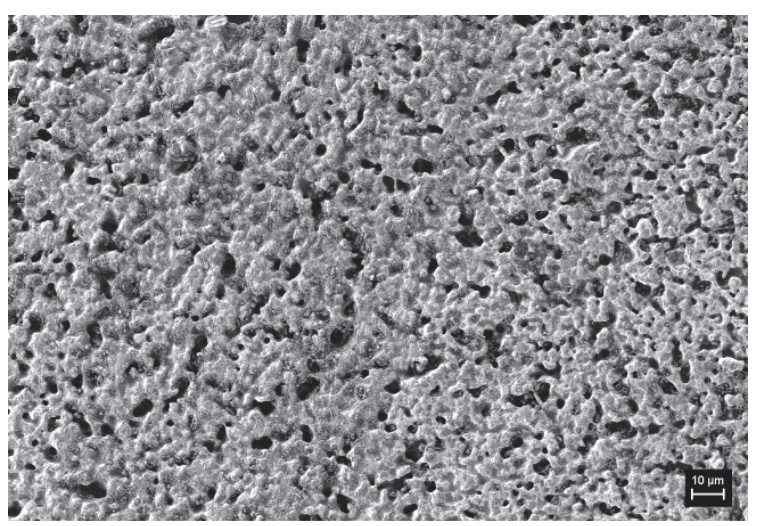

Abb. 3a. FESEM-Aufnahme der Oberfläche der PtGlas-Dickschichtstruktur, Referenzprobe.

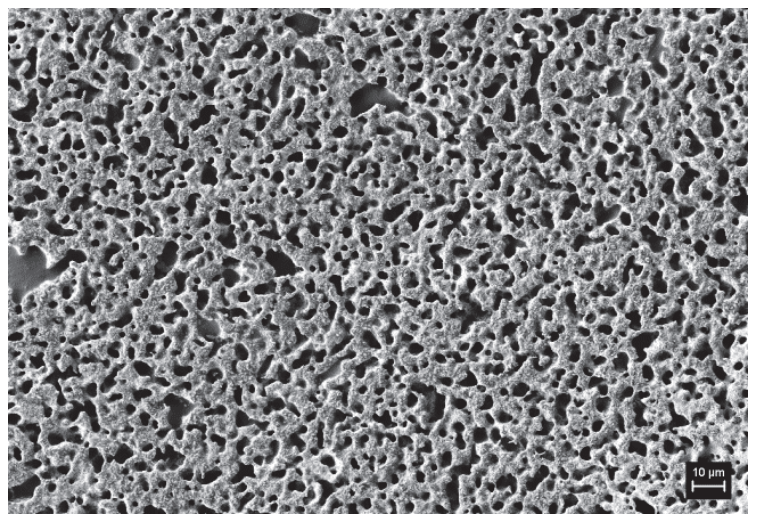

Abb. 3b. FESEM-Aufnahme der Oberfläche der PtGlas-Dickschichtstruktur, $\mathrm{HCl}$-Auslagerungsprobe.

Zerstörung der Glasphase zur Folge, was in der erhöhten Porosität und der damit einhergehenden stark verringerten mechanischen Stabilität der Dickschicht resultierte.

Die zusätzlich ausgelagerten Quarzglas- und Platinproben zeigten keinen messbaren Massenverlust und keine anhand von FESEMAufnahmen erkennbare Degradation.

Als Werkstoffe für die Sensorfertigung gingen damit 8YSZ, Quarzglas und Platin aus den Untersuchungen hervor.

\section{Sensoraufbau}

Die Heizelemente werden in keramischer Dickschichttechnologie gefertigt und bestehen aus einem $120 \mu \mathrm{m}$ dicken, planaren Substrat aus 8YSZ, auf welches mittels Siebdrucktechnologie Leitbahnstrukturen aus Platin8YSZ - Cermet aufgebracht werden. Die dem Cermet zugrunde liegende Dickschichtpaste wurde im Rahmen der Sensorentwicklung am Fraunhofer-IKTS entwickelt.

Zum Schutz der Leitbahnstrukturen vor unerwünschten korrosiven Wechselwirkungen mit der Gasatmosphäre, sind diese mit einer Schutzschicht aus 8YSZ-SiO ${ }_{2}-$ Komposit abgedeckt. In Abhängigkeit von Zusammensetzung und Wärmebehandlung des Komposits lassen sich so gasdichte Schutzschichten realisieren. Für eine zusätzliche katalytische Funktionalisierung können die Heizelemente mit einer Beschichtung aus nanoskaligen Platin-Partikeln versehen werden, welche im Aerosoldruckverfahren mit einer Dicke von $0,1 \ldots 1 \mu \mathrm{m}$ auf der Schutzschicht abgeschieden wird.

Die elektrische Kontaktierung der Heizelemente wird mit Hilfe eines Platindrahts realisiert, welcher mittels Sinterverbindung an den Kontaktflächen der Leitbahnstrukturen angebracht wird.

Die Heizelemente werden schließlich in einseitig offene Quarzglas-Trägerröhrchen eingepasst und an den Kontaktdrähten gasdicht eingeschmolzen. In der folgenden Abb. 4 ist ein solches Sensorelement dargestellt.

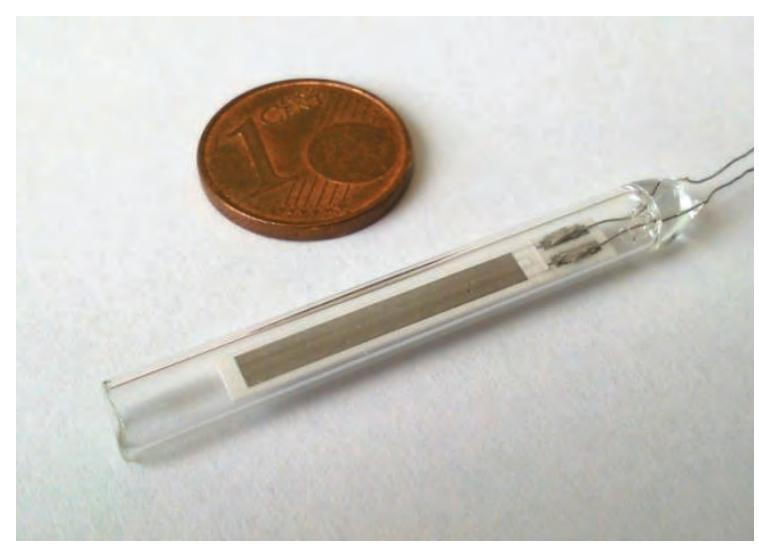

Abb. 4. Sensorelement: Kontaktiertes keramisches Heizelement mit Schutzschicht und PtFunktionsschicht in Quarzglas-Trägerröhrchen.

Für die Gasmessung werden vier Sensorelemente in ein geeignetes Gehäuse integriert und elektrisch in Form einer WheatstoneBrücke verschaltet. Dabei kommen je zwei Sensorelemente mit und zwei ohne katalytische Beschichtung zum Einsatz, die abgegriffene Brückenspannung ist schließlich proportional zur gesuchten Wasserstoffkonzentration.

\section{Messprinzip}

Die Funktion des Sensors beruht auf dem Wärmetönungsprinzip. Dabei ruft die am Heizelement beim kontrollierten Umsatz von Wasserstoff mit Chlor zu Chlorwasserstoff frei werdende Reaktionswärme eine Änderung der Heizertemperatur hervor. Diese Temperaturänderung hat wiederum eine messbare Änderung des elektrischen Widerstands der Heizerstruktur zur Folge, welche damit proportional zur Wasserstoffkonzentration im Messgas ist. Die für die Reaktion erforderliche Aktivierungsenergie wird von den Heizelementen bereitgestellt, die notwendige Temperatur liegt im Bereich von $300 \ldots 400^{\circ} \mathrm{C}$. Um stark 
exotherme Exkurse der Reaktion zu vermeiden welche zu einer Überhitzung und Zerstörung der Heizelemente führen können, wird die Reaktionsrate über zwei Mechanismen begrenzt: Zum einen wird der Gaszutritt zum Heizelement durch eine Diffusionsbarriere am Eingang des Sensorelements limitiert. Zum anderen können die Heizelemente mit einer gepulsten Versorgungspannung beheizt werden, wodurch die für die Reaktion notwendige Aktivierungsenergie nur für kurze Zeitintervalle bereitgestellt wird.

\section{Ergebnisse}

Die räumliche Temperaturverteilung auf den Heizelementen spielt eine entscheidende Rolle bei der Beurteilung der Leistungsfähigkeit und Langzeitbeständigkeit der Sensoren. Je homogener die Temperaturverteilung entlang der Heizbahnen, desto reproduzierbarer ist das Ansprechverhalten in der Gasatmosphäre. Im Gegensatz dazu stellen starke lokale Temperaturüberhöhungen aufgrund der damit verbundenen thermomechanischen Spannungen ein Risiko für die Langzeitstabilität der Sensorelemente dar. Es wurden daher Sensorelemente mit verschiedenen Versorgungsspannungen im Bereich $1 \ldots 10 \mathrm{~V}$ beaufschlagt und gleichzeitig mit einer hochauflösenden Infrarotkamera überwacht. In Abb. 5 ist beispielhaft eine solche Infrarotaufnahme bei Beaufschlagung mit $10 \mathrm{~V}$ Gleichspannung gezeigt. Die dargestellte Temperatur ist nicht als exakter Absolutwert, sondern nur als näherungsweise Größe zu verstehen, da die Messungen nicht entsprechend der Emissionseigenschaften des Materialsystems korrigiert wurden. In der Infrarotaufnahme zeigt sich jedoch die vergleichsweise hohe Homogenität der Temperaturverteilung entlang der Heizbahnen, gleichzeitig aber auch der starke Temperaturabfall auf dem Substratmaterial zwischen den Leiterbahnen sowie auf dem Substratrand.

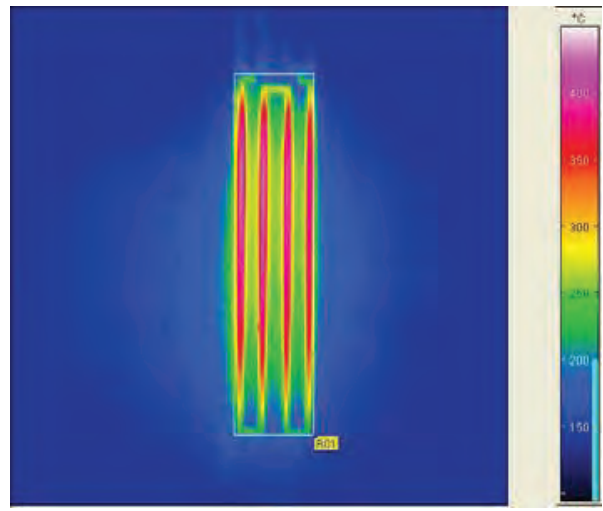

Abb. 5. Wärmebildaufnahme der Probenoberfläche bei Beaufschlagung mit $10 \mathrm{~V}$ Gleichspannung.
Zur Untersuchung des Ansprechverhaltens des Sensors wurde intervallweise Wasserstoff in einen Chlorgasstrom $\quad\left(80{ }^{\circ} \mathrm{C}, 100 \%\right.$ rel. Feuchte, $40 \mathrm{I}_{\mathrm{n}} / \mathrm{h}$ ) dosiert. Abb. 6 zeigt das Sensorsignal bei einer derartigen Messung. Die drei Hauptpeaks mit einer Signalamplitude von ca. $30 \mathrm{mV}$ entsprechen dabei der Sensorantwort auf eine $\mathrm{H}_{2}$-Konzentration von ca. 1 Vol.-\% im Messgas. Die Messergebnisse deuten an, dass mit dem Sensor auch wesentlich geringere Wasserstoffkonzentrationen detektierbar sind. Die Ansprechzeit $t_{90}$ beträgt im Falle der dargestellten Messwerte im Mittel $6 \mathrm{~s}$.

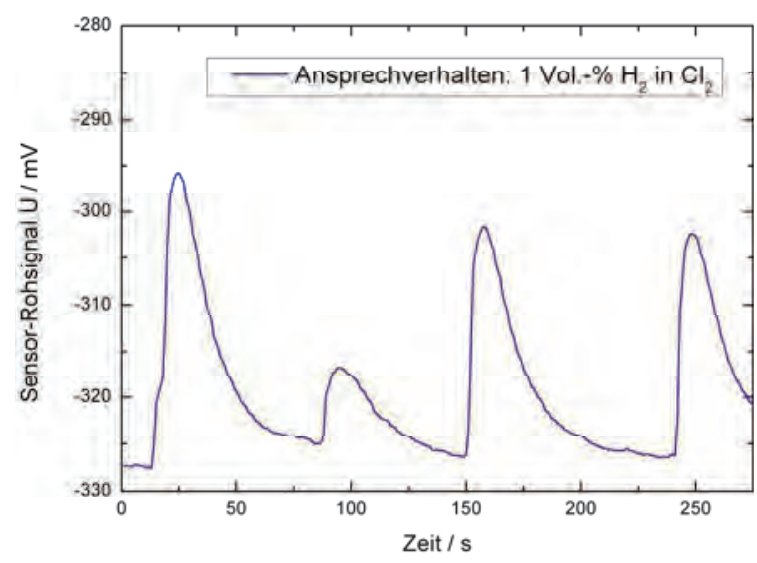

Abb. 6. Ansprechverhalten des Sensors auf 1 Vol.-\% Wasserstoff in Chlorgas.

Die Korrosionsbeständigkeit der Sensorelemente wurde in zahlreichen Auslagerungsuntersuchungen in $\mathrm{HCl}$-Dampf und Chlorgas nachgewiesen. Nach Auslagerung von sechs Sensorelementen in Chlorgas $\left(80^{\circ} \mathrm{C}, 100 \%\right.$ rel. Feuchte) über $1500 \mathrm{~h}$ lagen sowohl die relative Masseänderung mit $0,1 \%$ als auch die relative Änderung des elektrischen Heizerwiderstands mit 0,7\% im Rahmen der Messunsicherheit. FESEM-Aufnahmen der Probenoberfläche einer Referenzprobe (Abb. 7a) und einer in Chlorgas ausgelagerten Probe (Abb. 7b) lassen keine offensichtlichen Unterschiede

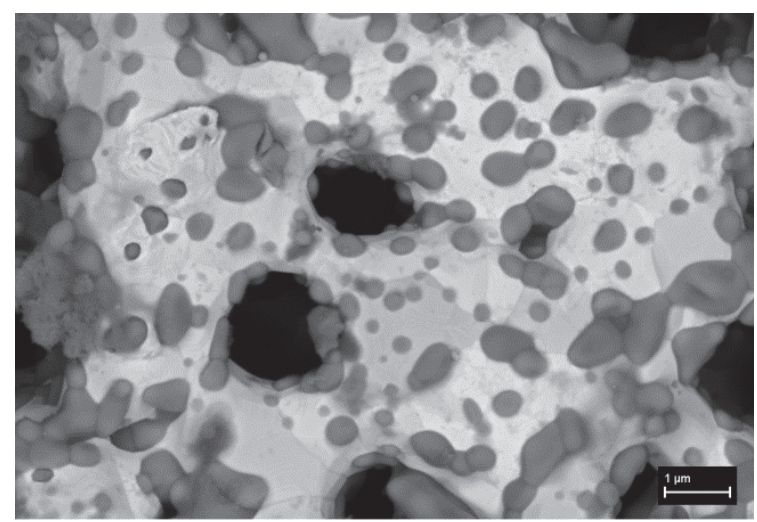

Abb. 7a. FESEM-Aufnahme der Oberfläche der Pt8YSZ-Dickschichtstruktur, Referenzprobe. 


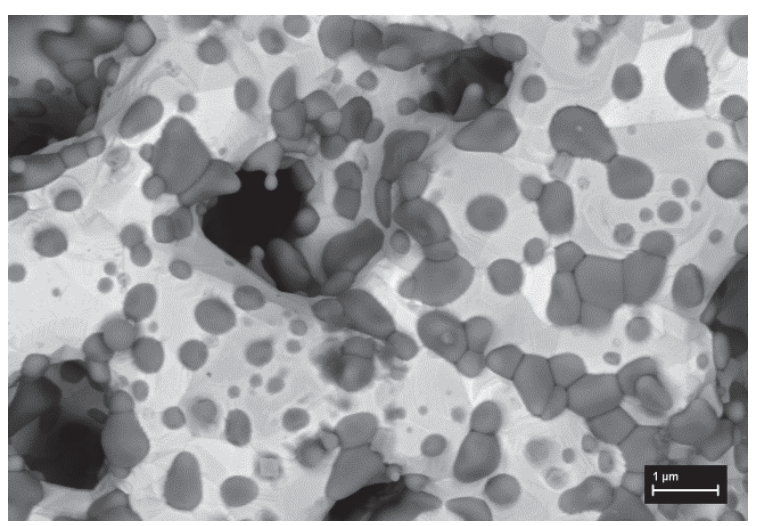

Abb. 7b. FESEM-Aufnahme der Oberfläche der Pt8YSZ-Dickschichtstruktur, Cl-Auslagerungsprobe.

in der Probenmorphologie erkennen: Sowohl Platin-Matrix (hellgrau) als auch 8YSZ-Partikel (dunkelgrau) weisen in beiden Fällen eine vergleichbare Struktur und Größe auf.

\section{Zusammenfassung}

Der für den Einsatz in der Chlorgasherstellung entwickelte Sensor beruht auf dem Wärmetönungsprinzip und erlaubt die Bestimmung von Wasserstoffkonzentrationen $\geq 1 \mathrm{Vol} .-\%$ in Chlorgas mit geringer Ansprechzeit. Kernelement des Sensors sind die in keramischer Dickschichttechnologie gefertigten Heizelemente, für welche neben dem Basiswerkstoff 8YSZ auch Quarzglas und Platin Verwendung finden. Diese Materialien zeigten in Voruntersuchungen eine hohe Korrosionsstabilität in $\mathrm{HCl}-\mathrm{Dampf}$ und feuchtem Chlorgas. In Verbindung mit der entwickelten Fertigungstechnologie erlauben sie den langzeitstabilen Betrieb des Sensors in feuchter heißer Chlorgasatmosphäre. Aufgrund des ausgezeichneten Ansprech- und Langzeitverhaltens ist der Einsatz des Sensors auch für andere Anwendungen denkbar, bei welchen der Wärmetönungseffekt in korrosiver Gasatmosphäre ausgenutzt werden kann.

\section{Literaturquellen}

[1] A. Paul, Chemical Durability of Glasses; A Thermodynamic Approach, Journal of Material Science 12, 2246-2268 (1977)

[2] M. Kord, V.K. Marghussian, B. Eftekhari-yekta, A. Bahrami, Effect of $\mathrm{ZrO}_{2}$ Addition on Crystallization Behaviour, Porosity and ChemicalMechanical Properties of a $\mathrm{CaO}-\mathrm{TiO}_{2}-\mathrm{P}_{2} \mathrm{O}_{5}$ Microporous Glass Ceramic, Materials Research Bulletin 44, 1670-1675 (2009); doi:10.1016/j.materresbull.2009.04.007
[3] G. Chen, J. Cheng, W. Chen, Effect of $\mathrm{Si}_{3} \mathrm{~N}_{4}$ on Chemical Durability of Chalcogenide Glass, Journal of Non-Crystalline Solids 220, 249-253 (1997)

[4] R. A. Rahimi, S.K. Sadrnezhaad, G. Raisali, Chemical Durability of Lead Silicate Glass in $\mathrm{HNO}_{3}, \mathrm{HCl}$ and $\mathrm{H}_{2} \mathrm{SO}_{4}$ Aqueous Acid Solutions, Journal of Non-Crystalline Solids 355, 169-174 (2009)

[5] M. Yoshimura, T. Hiuga, S. Somiya, Dissolution and Reaction of Yttria-Stabilized Zirconia Single Crystals in Hydrothermal Solutions, Journal of the American Ceramic Society 69 (7), 583-584 (1986)

[6] S. Lawson, Environmental Degradation of Zirconia Ceramics, Journal of the European Ceramic Society 15 (6), 485-502 (1995) 